\title{
Steady and Unsteady calculations on Thermal Striping Phenomena in Triple-Parallel Jet
}

\author{
Y.Q. Yu ${ }^{1}$, E. Merzari ${ }^{1}$, J.W.Thomas ${ }^{1}$, A. Obabko ${ }^{2}$, S. M. Aithal ${ }^{3}$ \\ 1: Nuclear Engineering Division, Argonne National laboratory, Lemont, IL 60439, United State \\ 2 : Mathematics and Computer Science Division, Argonne National laboratory, Lemont, IL \\ 60439, United State \\ 3 : Computing, Environment and Life Sciences Directorate, Argonne National laboratory, \\ Lemont, IL 60439, United State
}

yyu@anl.gov

\begin{abstract}
The phenomenon of thermal striping is encountered in liquid metal cooled fast reactors (LMFR), in which temperature fluctuation due to convective mixing between hot and cold fluids can lead to a possibility of crack initiation and propagation in the structure due to high cycle thermal fatigue. Using sodium experiments of parallel triple jets configuration performed by Japan Atomic Energy Agency (JAEA) as benchmark, numerical simulations were carried out to evaluate the temperature fluctuation characteristics in fluid and the transfer characteristics of temperature fluctuation from fluid to structure, which is important to assess the potential thermal fatigue damage. In this study, both steady (RANS) and unsteady (URANS, LES) methods were applied to predict the temperature fluctuations of thermal striping. The parametric studies on the effects of mesh density and boundary conditions on the accuracy of the overall solutions were also conducted. The velocity, temperature and temperature fluctuation intensity distribution were compared with the experimental data. As expected, steady calculation has limited success in predicting the thermal-hydraulic characteristics of the thermal striping, highlighting the limitations of the RANS approach in unsteady heat transfer simulations. The unsteady results exhibited reasonably good agreement with experimental results for temperature fluctuation intensity, as well as the average temperature and velocity components at the measurement locations.
\end{abstract}

Keywords: thermal striping, triple jets, URANS, LES 


\section{Introduction}

Fluctuating temperatures in the core outlet of sodium-cooled fast reactors (SFRs) may cause high-cycle thermal fatigue (a.k.a. thermal striping) in the structures in the upper plenum, eventually causing damage to important structures above the core. Jets expel from each of the core subassemblies into the outlet plenum (or hot pool), and the mixing from interacting, turbulent jets of a wide range of flow rates and temperatures results in highly complex flow and temperature evolutions. Nearby structures, including core instrumentation, control rod drivelines, and their supports, may see a range of fluctuating fluid temperatures during normal operation. Predicting this phenomenon, which is important for design and operation, is further complicated by the influence of the solid structure on the fluid temperature caused by the difference in heat capacity. Such code predictions need to be validated against relevant experiments before they can be used reliably in design studies or in supporting safety and licensing activities. Experiments using sodium are particularly useful, because its high thermal conductivity influences the time-scale of these fluctuations. Unfortunately, because sodium is opaque, simultaneous optical techniques of measuring high-resolution velocity profiles in sodium are not possible. Therefore, validating the velocity predictions must utilize experiments in transparent fluids like water and air.

The problem of thermal striping in the core outlet region of SFRs is of great interest to the international community. Wakamatsu et al. (1995) studied thermal striping experimentally with air, water and liquid sodium as a working fluid. It was found that the peak-to-peak amplitude of the surface temperature fluctuations was about half of that of the fluid around the solid plate. Large temperature fluctuations were measured in Nam and Kim's (2004) experiment with various combinations of inlet velocity and temperature. The experimental studies have provided valuable information in understanding thermal striping. But it has been limited to relatively simple geometries. There has been a continual effort to predict the temperature fluctuations in thermal striping using numerical simulations. Many simulations with varying degrees of success in terms of predicting mean velocity and thermal fields have been performed (Choi et al. (2005), Choi and Kim (2009)). As expected, it is found that RANS models are not able to predict the temperature fluctuations correctly. Therefore, URANS and large eddy simulation (LES) technique has been successfully used in limited areas of nuclear applications to investigate unsteady flow and thermal fluctuations. A few examples of these simulations for calculating turbulent flows and heat transfer in the nuclear field are given in Cheng and $\mathrm{Yu}$ (2009) and Yu et al., (2011). The JAEA cites early work for thermal striping by Wood (1980), Brunings (1982), Betts et al. (1983), Moriya et al.(1991) and Tokuhiro et al. (1998) in their reports. In CEA, Tenchine and Nam (1987), Tenchine and Moro (1997) investigated the mixing behavior of coaxial jets of sodium and compared the results of sodium with air jets experiment. They found that the air tests can be used to predict the thermal fluctuation behavior in the sodium reactor. In the DOE complex, the most recent experiments have been performed using Argonne's coaxial air-jet mixing facility MAX, which employs the high-resolution optical measurement techniques for the velocity distribution, along with wall surface temperature measurements via thermal imaging and within-jet temperature profile measurements via fiber optics (Merzari (2010), Lomperski et al. (2012)). 
Given the importance of validating thermal striping predictions to the international community, and the value of high-quality measurements of the phenomenon in sodium, the members STC-A7 collaboration on Sodium-Cooled Fast Reactor Thermal-Hydraulics decided to initiate a benchmark exercise using the JAEA experiment, named PLAJEST, which is part of a larger series of related experiments in turbulent jet mixing. Tokuhiro et al. (1997), Tokuhiro and Kimura (1999), Kimura et al. (1997, 2007) performed a water experiment of the triple-parallel jets mixing phenomena called WAJECO and evaluated the mixing process among the jets. The attenuation process of the temperature fluctuation from fluid to structure was very important to predict the thermal striping phenomena. Kimura et al. (2007) performed a sodium experiment (called PLAJEST) that had triple-parallel jets along a stainless steel wall and showed that the transfer characteristics of temperature fluctuation from fluid to structure could be evaluated by using a heat transfer coefficient obtained from a transfer function between temperature fluctuations in fluid and structure. Furthermore, in the water experiment (WAJECO) with the same configured test section in the sodium experiment (PLAJEST), a stainless steel plate with thermocouples was set along flow in order to evaluate the characteristics of temperature fluctuation transfer from fluid to structure compared with that in the sodium experiment (Kimura et al.(2005,2008)).

This paper provides a description of the PLAJEST benchmark problem. Furthermore, both steady (RANS) and unsteady (URANS, LES) calculations were applied to predict the thermal striping phenomenon. The benchmark exercise in this paper is being performed under the auspices of an international collaboration on thermal hydraulics for sodium-cooled fast reactor development with participation from the Japan Atomic Energy Agency, the U.S. Department of Energy, and the France's Commissariat a l'energie atomique et aux energies alternatives.

\section{Description of PLAJEST Experiment and Benchmark specifications}

(a)

Figure 3-1 shows a schematic of the test section of PLAJEST. The $x$ - axis corresponds to the depth direction normal to the vertical wall, the $y$-axis is the horizontal direction in which the jets are aligned, and the $z$-axis is vertical direction in which flow is injected. The origin is set at the center of the cold jet on the nozzle outlet and on the wall surface. In the test section, a rectangular metal block with slope on both sides is installed in the bottom of the test section. The metal block has three discharged nozzle outlets on the top surface. The height of the block is 85 $\mathrm{mm}$ from the bottom of the test section. The cross section of each nozzle outlet is rectangular. Width in the horizontal direction is $20 \mathrm{~mm}$ and nozzle length in depth direction is $180 \mathrm{~mm}$. A cold jet vertically flows out from the center nozzle and the hot jets vertically flow out from the nozzle outlets on both sides of the center. The test section is placed between two vertical plates. The parallel plates are installed on both sides of the nozzle block in the x-direction. The SS316 plate at $x=0$, is instrumented to investigate temperature fluctuation in the structure.

Equations 2-1 through 2-4 follow those in Kimura (2007)

Table 2-1 shows typical experimental conditions as candidates for the benchmark exercise. The Reynolds number is defined as follows:

$$
R e=\frac{V_{m} D}{v}
$$




$$
V_{m}=\frac{V_{c}+2 V_{h}}{3}
$$

D is a nozzle width $(=20 \mathrm{~mm})$ and $V_{m}$ is mean discharged velocity of three jets. $V_{c}$ is the mean velocity of the cold jet from the center nozzle and $V_{h}$ is the mean velocity of the hot jets from the nozzles on both sides. The discharged temperature difference, $\Delta \mathrm{T}$, is defined as follows:

$$
\Delta \mathrm{T}=\mathrm{T}_{\mathrm{h}}-T_{c}
$$

$T_{h}$ and $T_{c}$ are time-averaged temperatures at the nozzle of the hot and cold jet respectively. Since the temperature difference is not so large, dependency of thermal property is not considered in the estimation of mixed-mean temperature $T_{m}$ as:

$$
T_{m}=\frac{V_{c} T_{c}+2 V_{h} T_{h}}{3 V_{m}}
$$

Equations 2-1 through 2-4 follow those in Kimura (2007)

Table 2-1 Experimental Conditions

\begin{tabular}{|c|rr|r|r|r|r|r|}
\hline \multirow{2}{*}{ Case } & \multicolumn{2}{|c|}{ Outer-slits/Hot Jets } & \multicolumn{2}{|c|}{ Center-slit/Cold Jet } & \multicolumn{3}{c|}{ Mixture } \\
& $V_{h}(m / s)$ & \multicolumn{1}{c|}{$\mathrm{T}_{\mathrm{h}}\left({ }^{\circ} \mathrm{C}\right)$} & \multicolumn{1}{|c|}{$V_{c}(\mathrm{~m} / \mathrm{s})$} & \multicolumn{1}{|c|}{$\mathrm{T}_{\mathrm{c}}\left({ }^{\circ} \mathrm{C}\right)$} & $V_{m}(\mathrm{~m} / \mathrm{s})$ & \multicolumn{1}{c|}{$\Delta \mathrm{T}\left({ }^{\circ} \mathrm{C}\right)$} & $\mathrm{T}_{\mathrm{m}}\left({ }^{\circ} \mathrm{C}\right)$ \\
\hline $\mathrm{A} 1$ & 0.51 & 347.5 & 0.51 & 304.5 & 0.51 & 43 & 333.2 \\
\hline $\mathrm{A} 2$ & 0.48 & 40.3 & 0.48 & 32.0 & 0.48 & 8.3 & 37.5 \\
\hline $\mathrm{B} 1$ & 0.51 & 349.8 & 0.32 & 311.0 & 0.45 & 38.8 & 340.5 \\
\hline
\end{tabular}

The iso-velocity case (Case A1) in which a characteristic dominant frequency was observed in the fluid temperature fluctuation around the center jet in the mixing region with stainless steel plate are selected as a candidate in benchmark exercise.

\section{Numerical Methodology}

\subsection{Computational Domain and Mesh Generation}

(a)

Figure 3-1 (b) shows the computational domain of $198 \mathrm{~mm}(=180 \mathrm{~mm}+18 \mathrm{~mm})$ in the $X$ direction, $500 \mathrm{~mm}$ in the $Y$ - direction and $685 \mathrm{~mm}(=600 \mathrm{~mm}+85 \mathrm{~mm})$ in the $Z$ direction from the bottom of the test section. (a)

Figure 3-1(a) also provides a numbering scheme for the outer boundaries, which will be referenced in the description of the boundary condition sensitivity studies. The figure also indicates a smaller region used to visualize the results. This domain is enlarged three times in the Y-direction for the purpose of choosing boundary condition, which is further discussed in section 3.2 .

Two planes and two lines in the computational domain are identified where code predictions for the velocity and temperature distribution have been extracted in later plots. They are referenced as the center plane, the near-wall plane, the centerline and the near-wall line in the 
following content. The center plane $(x=85 \mathrm{~mm})$ is in the middle of two partition plates and the near-wall plane $(x=1 \mathrm{~mm})$ is in the vicinity of one partition plate. The two lines are on the two planes respectively at $z=100 \mathrm{~mm}$ from the nozzle exit in the vertical position where the main convective mixing occurred. All the velocity components are normalized with the discharge velocity, $V_{\text {exil }}=0.51 \mathrm{~m} / \mathrm{s}$. The temperature is normalized by the following equation:

$$
T^{*}=\frac{\left(T-T_{c}\right)}{\Delta T}
$$

where $T^{*}$ is non-dimensional temperature, $\Delta \mathrm{T}$ is the temperature difference between hot jet and cold jet.

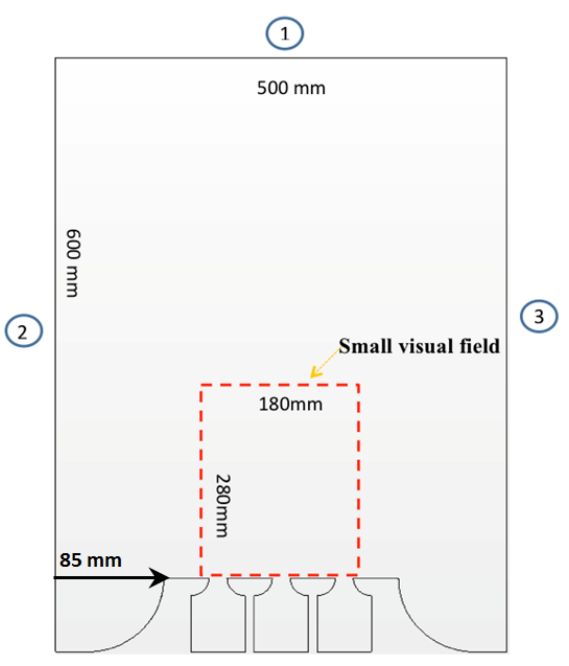

(a)

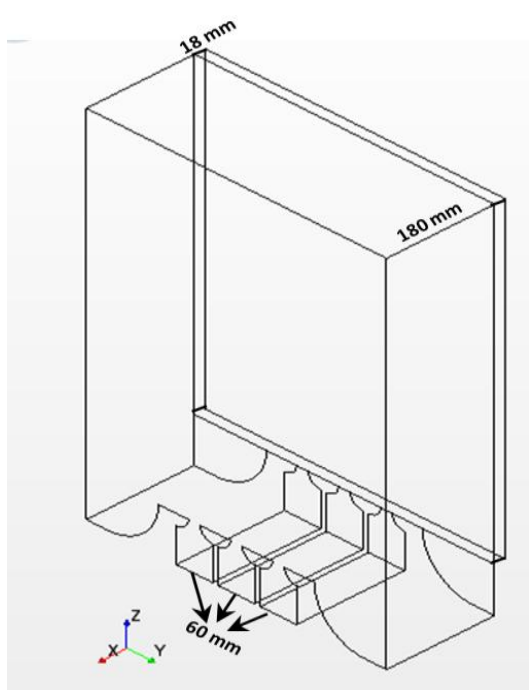

(b)

Figure 3-1. Computational Domainfor the Model of the PLAJEST Benchmark experimental set-up (a) 2-D plane showing BCs (b) 3-D showing axes

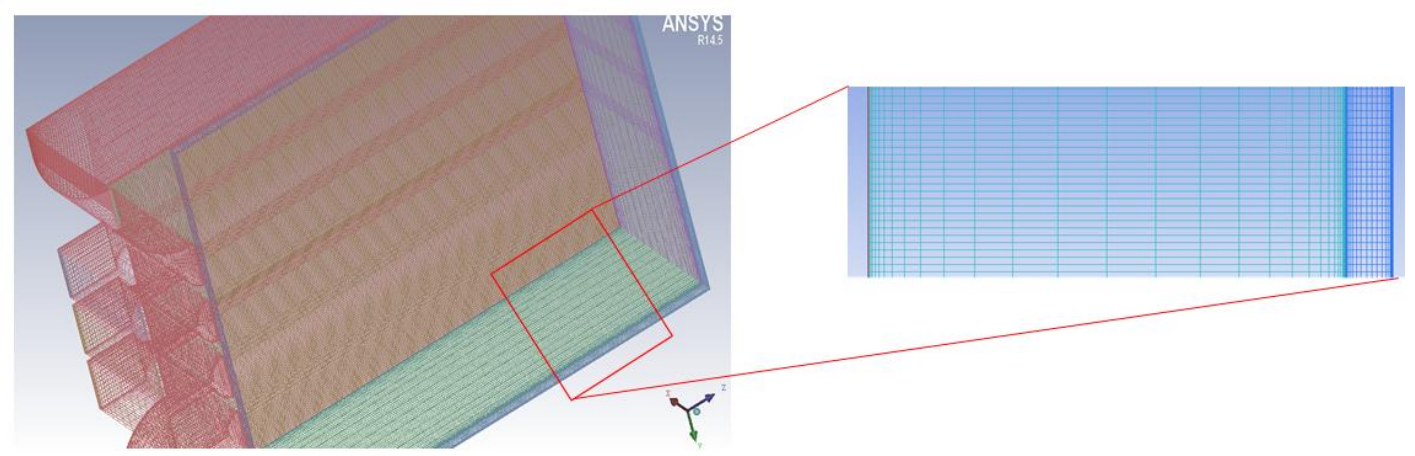

Figure 3-2. Hexahedral Mesh Structure 

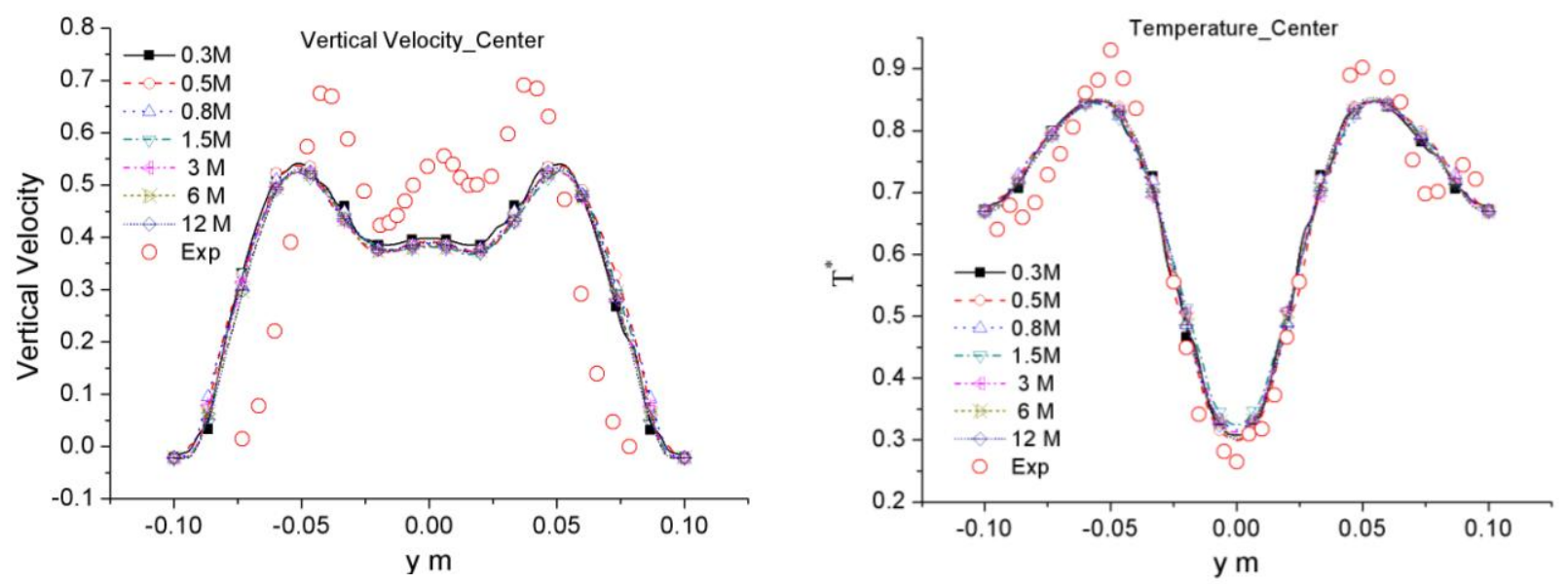

Figure 3-3. Results of mesh refinement study: velocity (left) and temperature(right)profiles

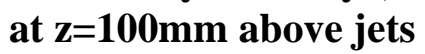

A purely hexahedral mesh (Figure 3-2) is used in this benchmark study. A mesh sensitivity study was performed to ensure $\mathrm{y}^{+}<1$ at the wall. The number of cells in the various meshes ranges from 0.3 million to 12 million. The effect of the number of cells on the velocity and temperature distribution is shown in Figure 3-3. It is seen that the solution is independent of mesh resolution in this range $(0.3 \mathrm{M}$ to $12 \mathrm{M})$. In order to obtain a reasonable resolution of the simulation, a mesh of 3 million cell is chosen for the RANS analysis. A refined mesh was generated for the URANS and LES analysis, primarily motivated by the mesh requirements for LES. A detailed description of the mesh is listed in Table 3-1.

Table 3-1. STAR-CCM+ Mesh Description

\begin{tabular}{llccc}
\hline & Horizontal & $\begin{array}{c}\text { Normal Direction } \\
\text { to wall }\end{array}$ & Vertical \\
\hline \multirow{2}{*}{ Coarse Mesh for steady-state RANS } & \multicolumn{3}{l}{} \\
\hline \multirow{2}{*}{ Fluid } & Number of Mesh & 250 & 35 & 330 \\
\cline { 2 - 5 } & Minimum of Mesh Size & $2 \mathrm{~mm}$ & $0.08 \mathrm{~mm}$ & $2 \mathrm{~mm}$ \\
\hline \multirow{2}{*}{ Refined Mesh for URANS and LES } & \multicolumn{3}{c}{50} \\
\hline \multirow{2}{*}{ Fluid } & Number of Mesh & 364 & 55 & 393 \\
\cline { 2 - 5 } & Minimum of Mesh Size & $2 \mathrm{~mm}$ & $0.08 \mathrm{~mm}$ & 343 \\
\hline \multirow{2}{*}{ Structure } & Number of Mesh & 364 & 38 & $2 \mathrm{~mm}$ \\
\cline { 2 - 5 } & Minimum of Mesh Size & $2 \mathrm{~mm}$ & $0.05 \mathrm{~mm}$ & \\
\hline
\end{tabular}

\subsection{Boundary Conditions}

A sensitivity study was performed to investigate the influence of various boundary conditions on the accuracy of the overall solution.. In this study, steady-state RANS simulations were performed, and the solid walls were not considered. The boundary conditions applied to the three boundaries are labeled symmetry $(\mathrm{S})$, outlet $(\mathrm{O})$, flow-split 


\section{$(\mathbf{F})$, and periodic (P), according to the ordering indicated in (a)}

Figure 3-1 (a).

(b)

Since it's hard to determine the fraction of the flow exit from the side boundary experimentally, a simulation wherein the computational domain was three times wider in horizontal direction (shown in Figure3-4) was performed to provide reference data. The deviations of the flow fraction exiting from the side boundary by using various typical boundary conditions are listed in Table 3-2.

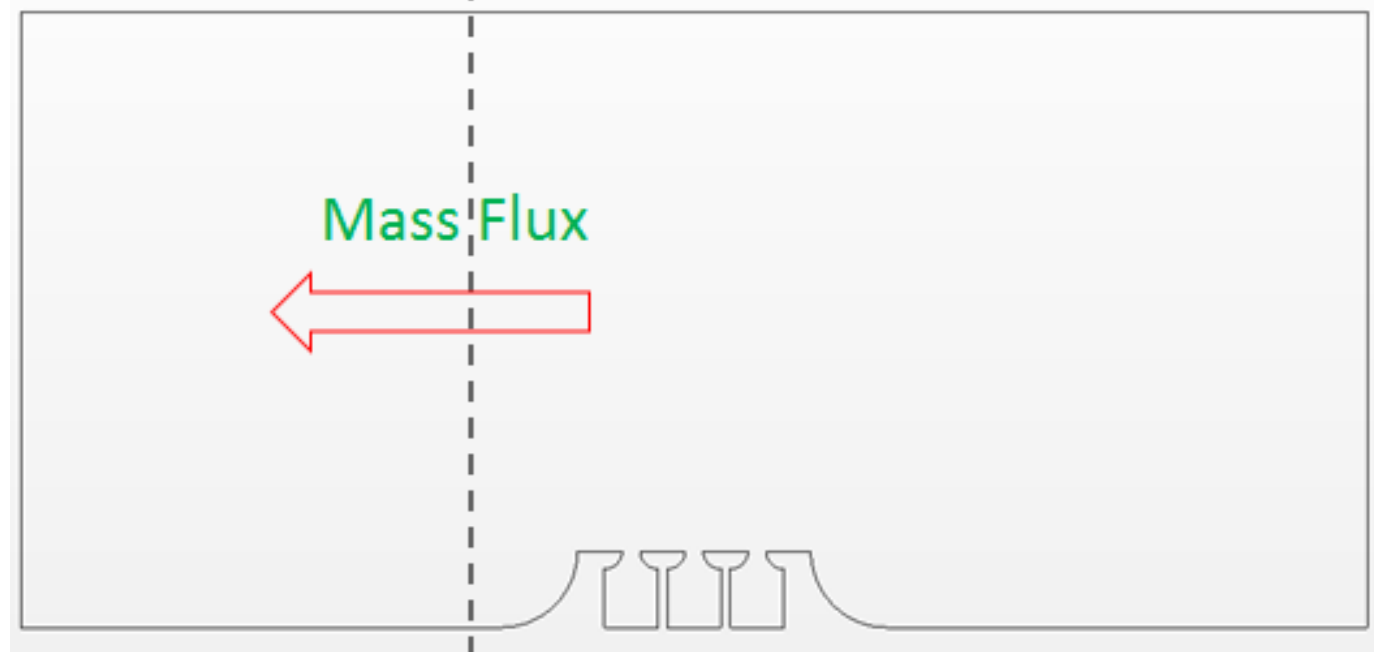

Figure3-4. Extensional computational domain

Table 3-2. Flow fraction exiting from the side boundary

\begin{tabular}{cccccccc}
\hline & FOO & FPP & FSS & OSS & OPP & OOO & Reference \\
\hline Fraction & $6.5 \%$ & $10.4 \%$ & 0 & 0 & $52.1 \%$ & $31.3 \%$ & $25.8 \%$ \\
Deviation & $19.3 \%$ & $15.4 \%$ & $25.8 \%$ & $25.8 \%$ & $-26.3 \%$ & $-5.5 \%$ & 0 \\
\hline
\end{tabular}

Velocity vectors on the center plane for various BCs described above are shown in Figure 3-5. These velocity vectors provide a better understanding on the effect of the boundary condition on the flow-field. In general, the effect of the boundary condition on the flow field in the small visual field (shown in Figure 3.1 (a)) is negligible since the boundary of the whole computational domain is sufficiently far from the small visual field. However, the boundary condition does have an effect on the flow distribution outside the small visual field, especially in the region near the boundary, as expected. In the FOO case, most flow exits from the top; only a small amount of fluid exit from the sides. In the FSS case, two symmetric vortexes appear on both side of the main flow stream; no flow exits or enters the domain from the sides due to the symmetry boundary condition. In the OOO case, flow exit from the top and the sides evenly with no vortex observed. Given these observations, symmetry boundary condition is not recommended if the computational cost is affordable because it adds some restrains on the flow near the boundary and may cause artificial recirculation of the flow. Based on the quantitative 
analysis, OOO is taken as the best combination. This boundary condition option was also applied to the URANS and LES analysis.

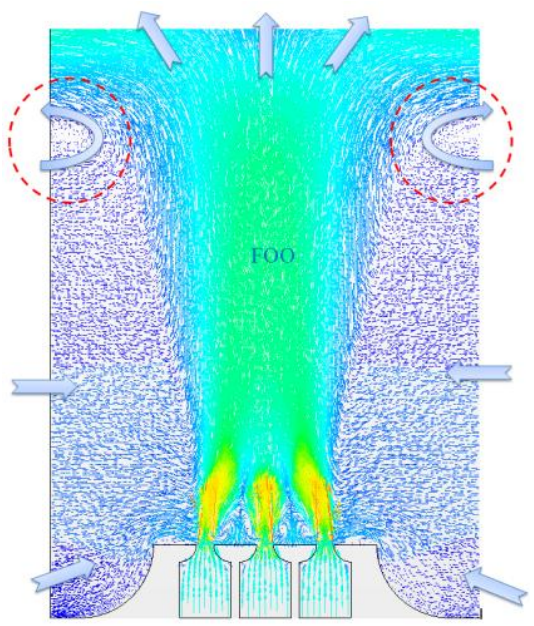

FOO

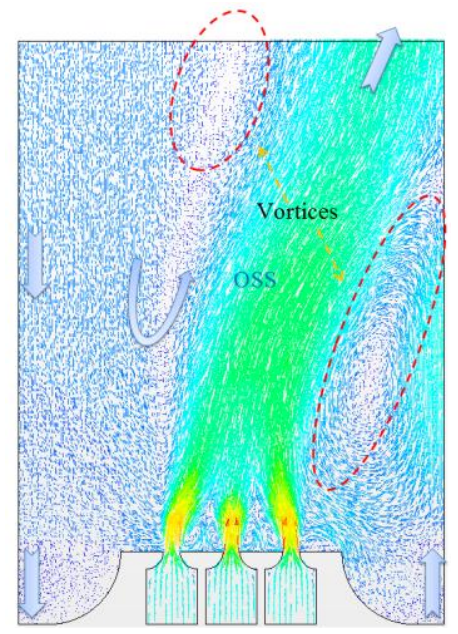

OSS

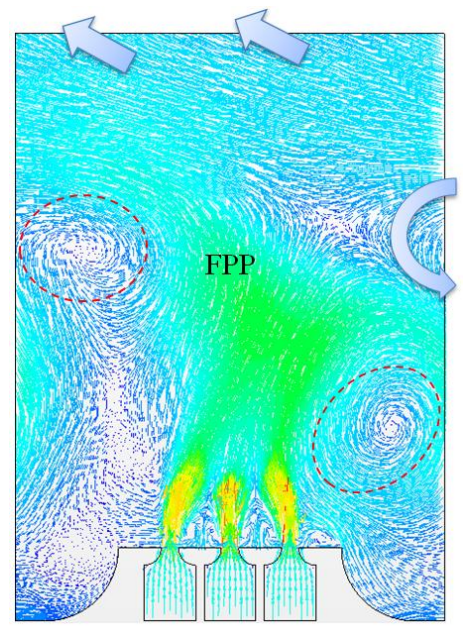

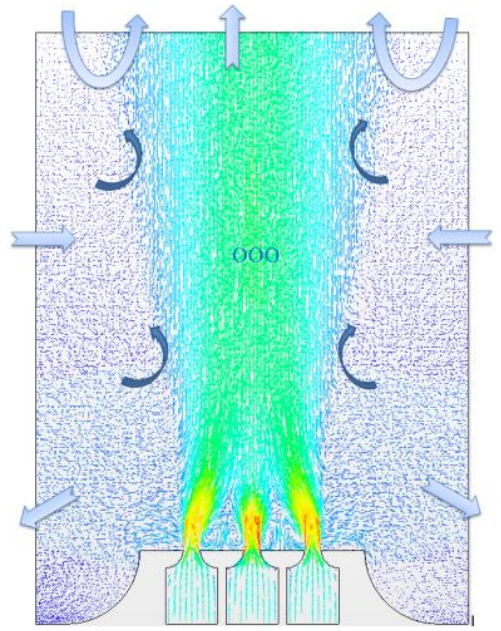

$\mathrm{OOO}$

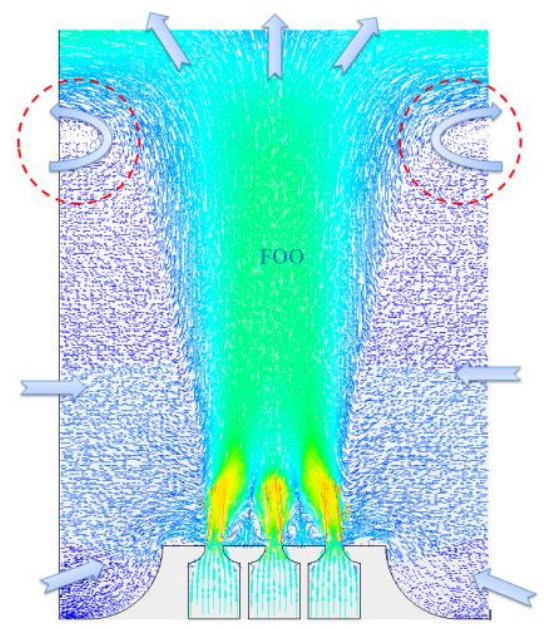

FOO

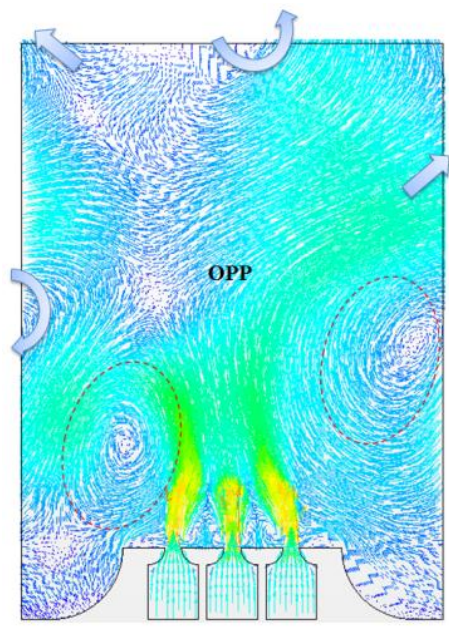

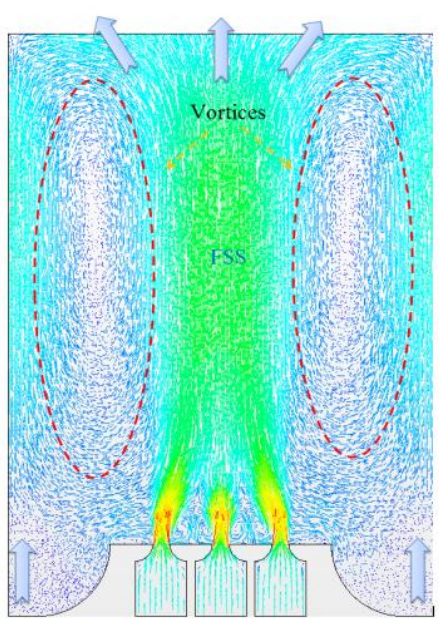

FSS

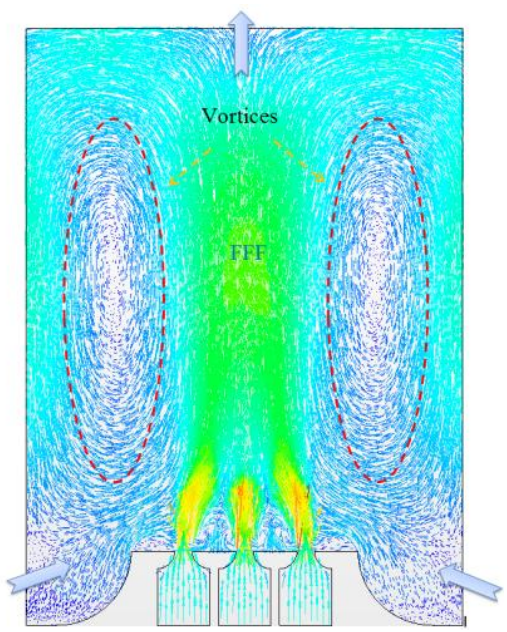

FSS 
Figure 3-5. Velocity Vector on the Center Plane

\section{Results and Discussions}

\subsection{Instantaneous Quantities}

The velocity and temperature fluctuations predicted with URANS model are presented in Figure $4-1$. The velocity ranges from $0.14 \mathrm{~m} / \mathrm{s}$ to $0.36 \mathrm{~m} / \mathrm{s}$ while temperature ranges from $311^{\circ} \mathrm{C}$ to $343^{\circ} \mathrm{C}$. Figure 4-2 shows the instantaneous temperature fluctuations in of the flow-field. The hot jets on both sides periodically lean to the cold jet, which results in the formation of some low temperature region. With the movement of these cold regions, the heat transfer between the fluids within these cold regions and the ambient hot fluid occur.

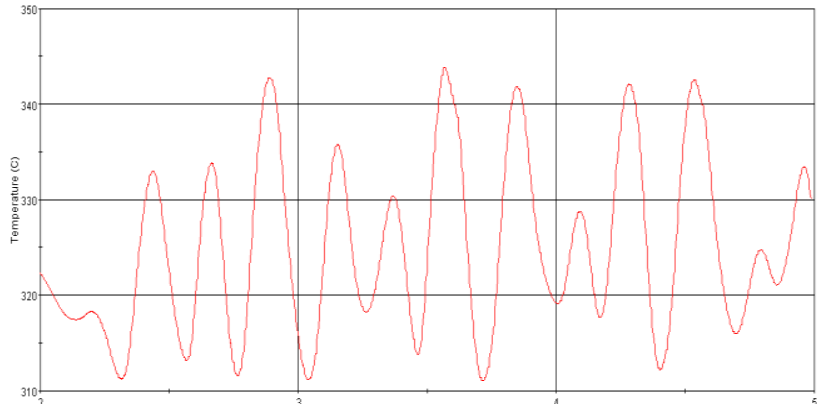

(a) Temperature fluctation

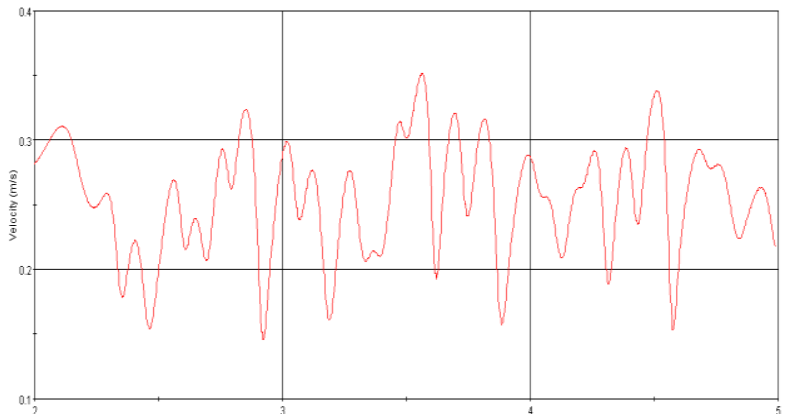

(b) Velocity fluctation

Figure 4-1. Temperature and Velocity fluctuation in URANS model

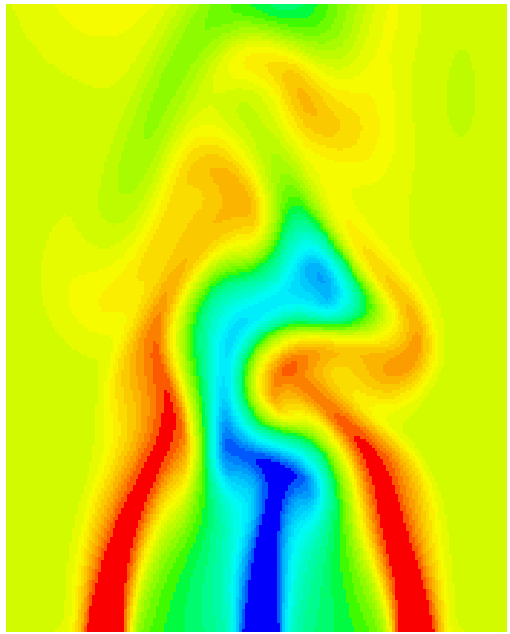

$10 \mathrm{~s}$

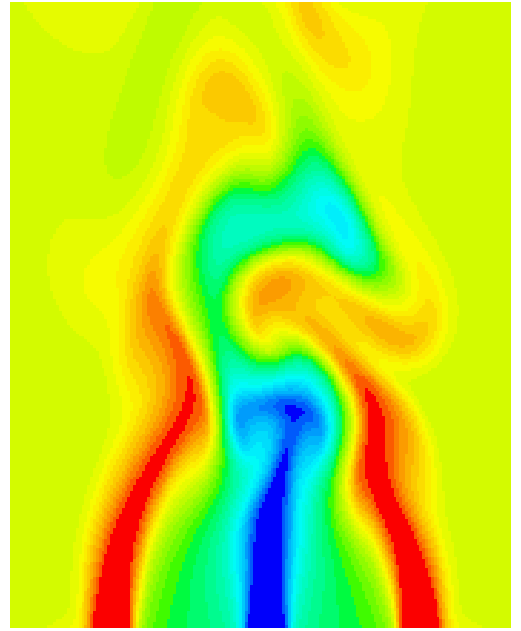

$10.1 \mathrm{~s}$

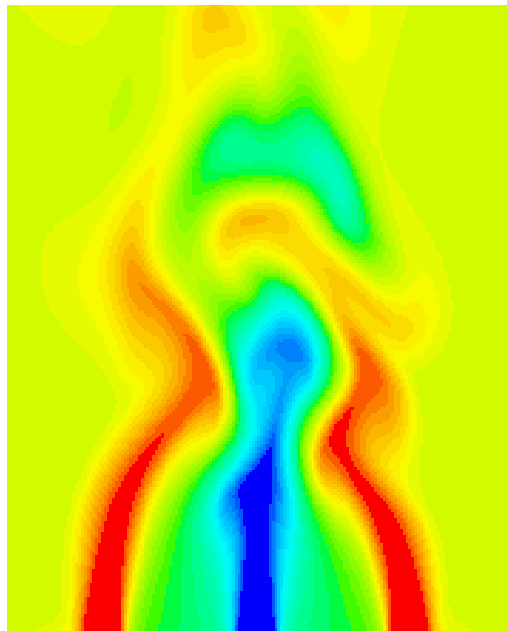

$10.2 \mathrm{~s}$ 


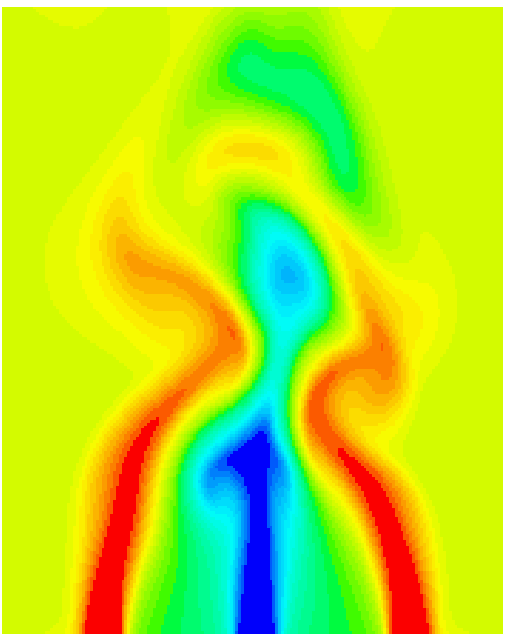

$10.3 \mathrm{~s}$

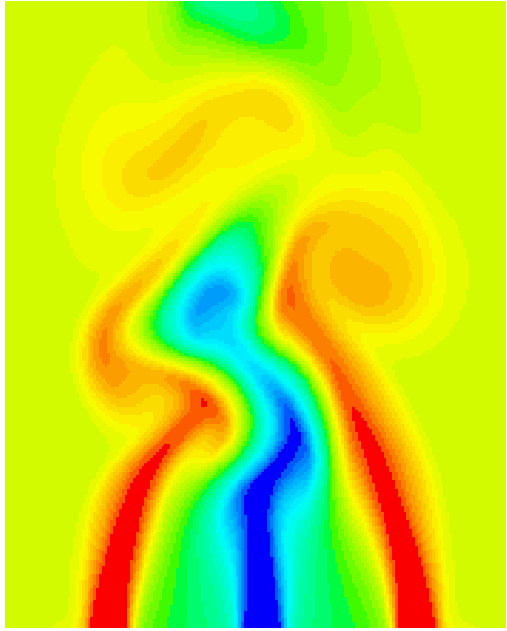

$10.6 \mathrm{~s}$

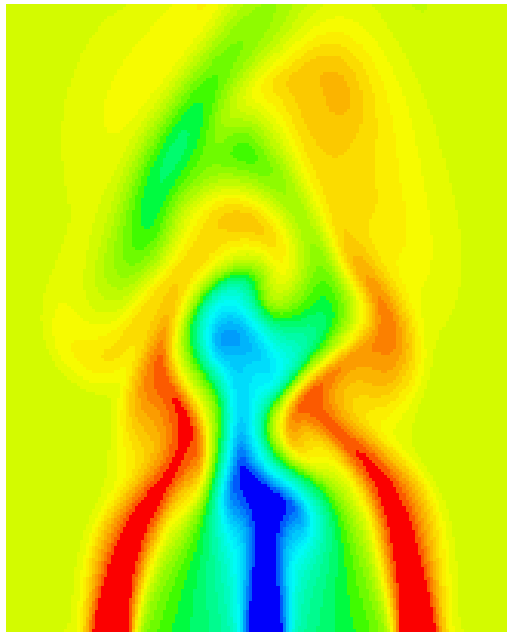

$10.9 \mathrm{~s}$

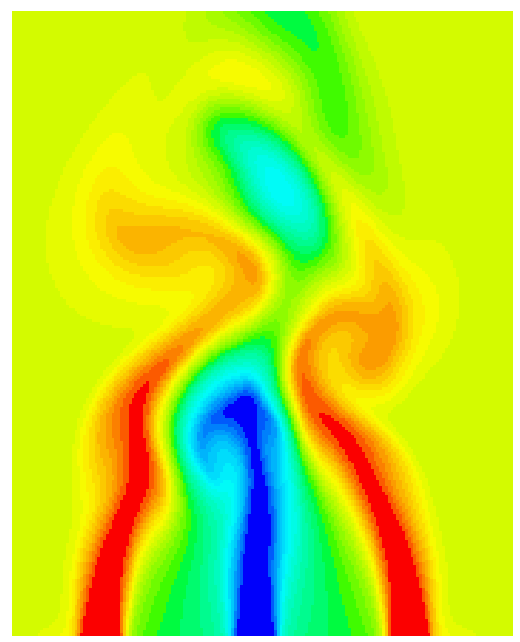

$10.4 s$

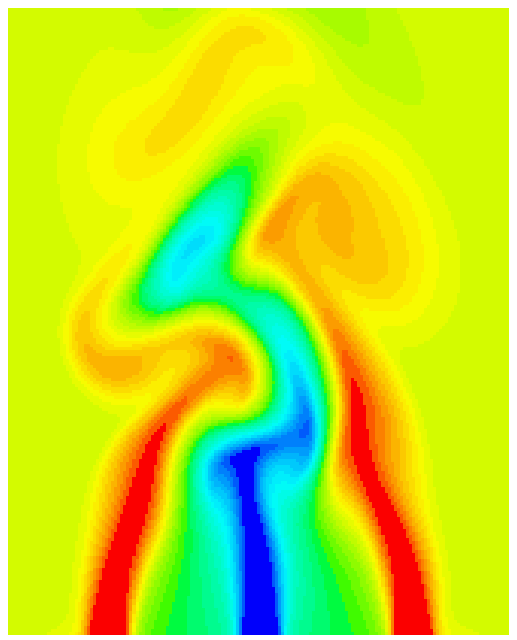

$10.7 \mathrm{~s}$

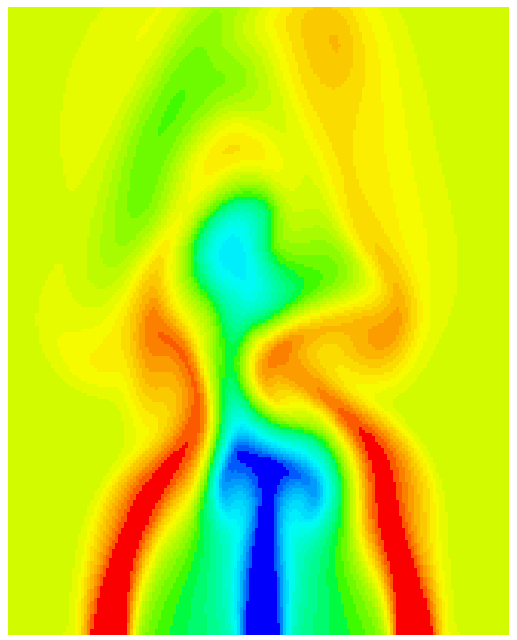

$11 \mathrm{~s}$

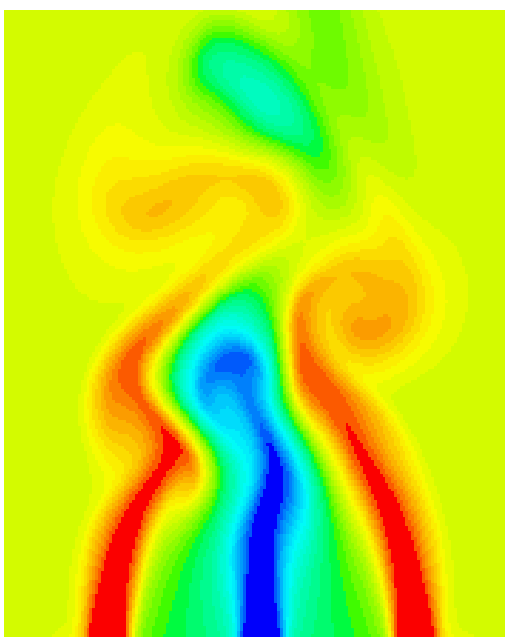

$10.5 \mathrm{~s}$

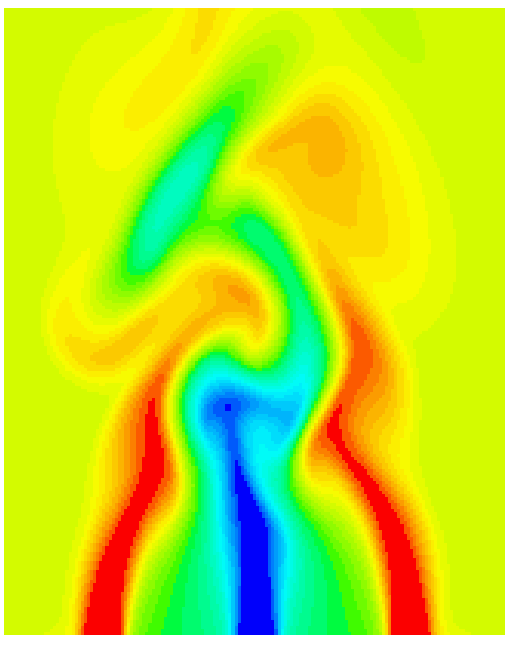

$10.8 \mathrm{~s}$

Figure 4-2. Temperature Contour on the Center Plane at Different Time Instance 


\subsection{Time-averaged Quantities}

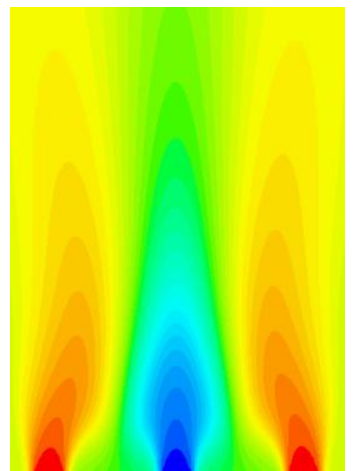

RANS

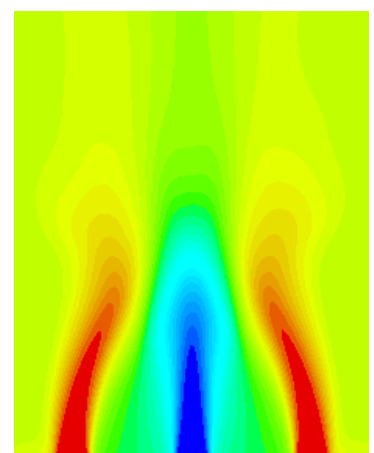

URANS

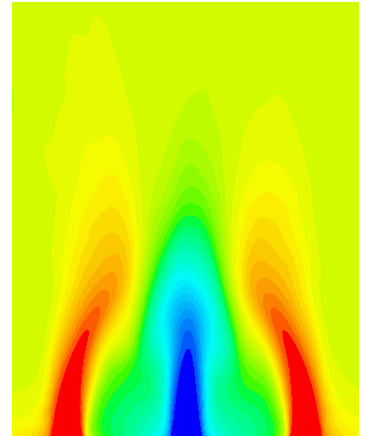

LES

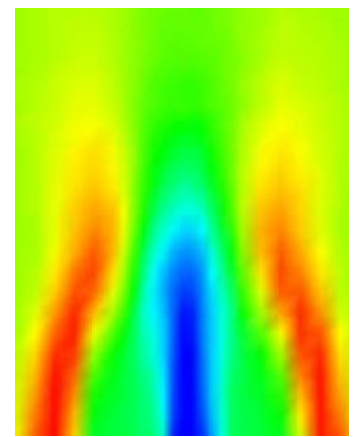

$\operatorname{Exp}$

Figure 4-3. Time-averaged Temperature Contour on the Center Plane

Figure 4-3 presents the time-averaged temperature distribution on the center plane with RANS, URANS and LES model. The shapes of the cold region (in blue) and the hot region (in red) with both URANS and LES model achieve good agreements with the experiment.

In Figure 4-4, a comparison of the time-averaged temperature fluctuation intensity (TFI) on the center plane between URANS, LES and experiment was shown. Temperature fluctuation intensity, TFI, is defined as a standard deviation of temperature as follows:

$$
T F I=\sqrt{\frac{1}{N} \sum_{i=1}^{N}\left(T_{i}-T_{\text {avg }}\right)^{2}}
$$

Moreover, the normalized temperature fluctuation intensity is as follows:

$$
T F I^{*}=\frac{T F I}{\Delta T}
$$

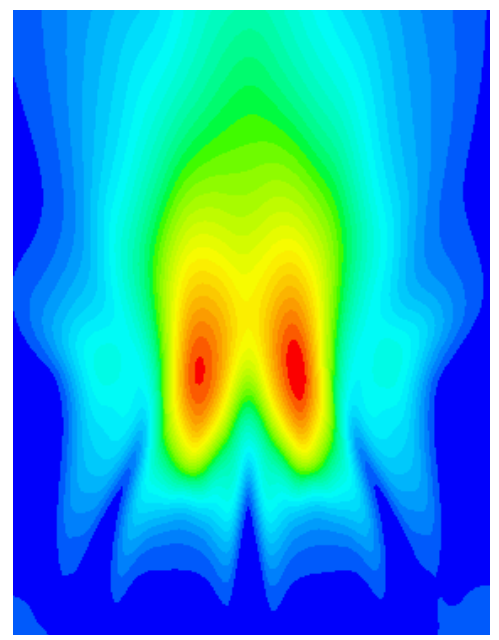

URANS

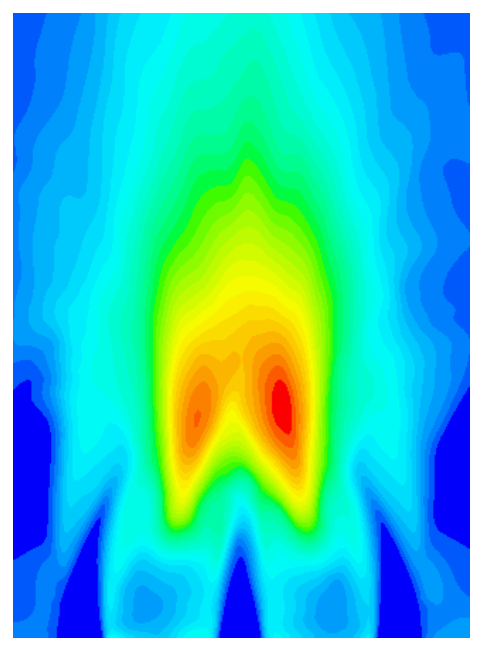

LES

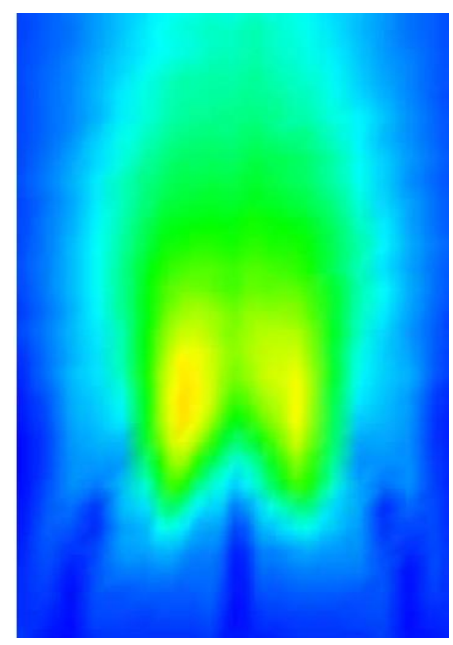

$\operatorname{Exp}$

Figure 4-4. Contour of Temperature Fluctuation Intensity on the Center Plane 
The contour pattern looks like a "butterfly", which agrees well with the experiment. Both URANS and LES model overestimate the TFI at $\mathrm{z}=80 \sim 120 \mathrm{~mm}$ vertically and in the region between the hot and the cold jest horizontally.

Figure 4-5 shows the comparison of the temperature distribution, velocity component and temperature fluctuation intensity on the centerline and the near-wall line between RANS, URANS and LES models. Remarkable improvements on the prediction are achieved with URANS and LES model as compared to the RANS models. The LES model predicts the experiment better than the URANS model does, especially on the TFI near the wall, which is significant to evaluate the thermal fatigue.

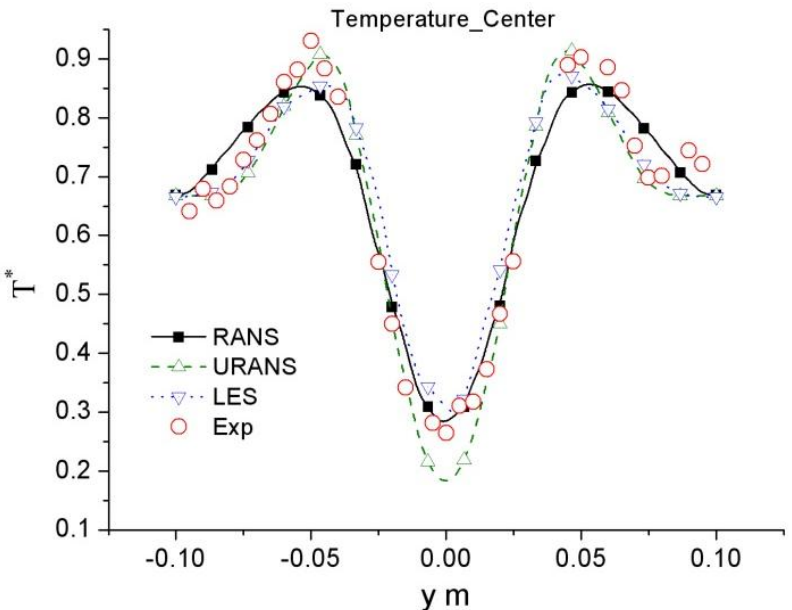

(a) Temperature along the centerline

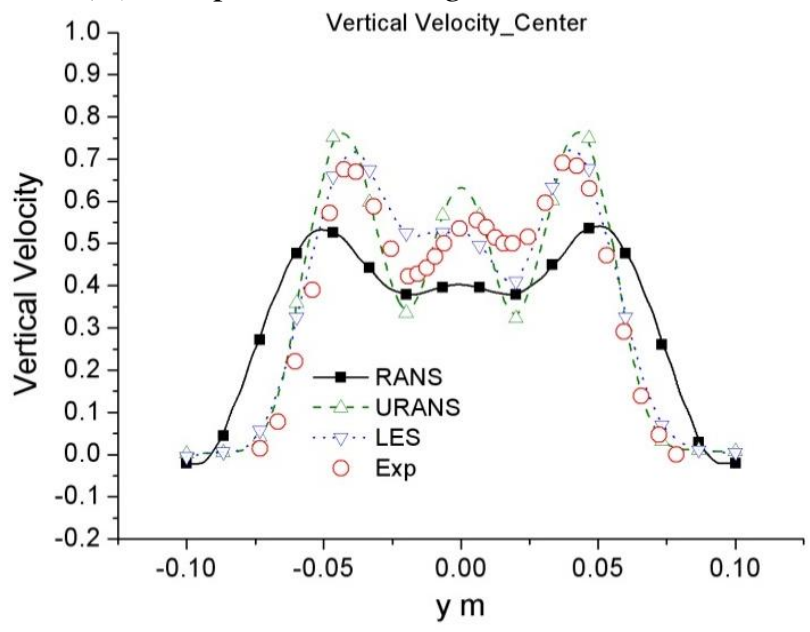

(c) Vertical Velocity along the centerline

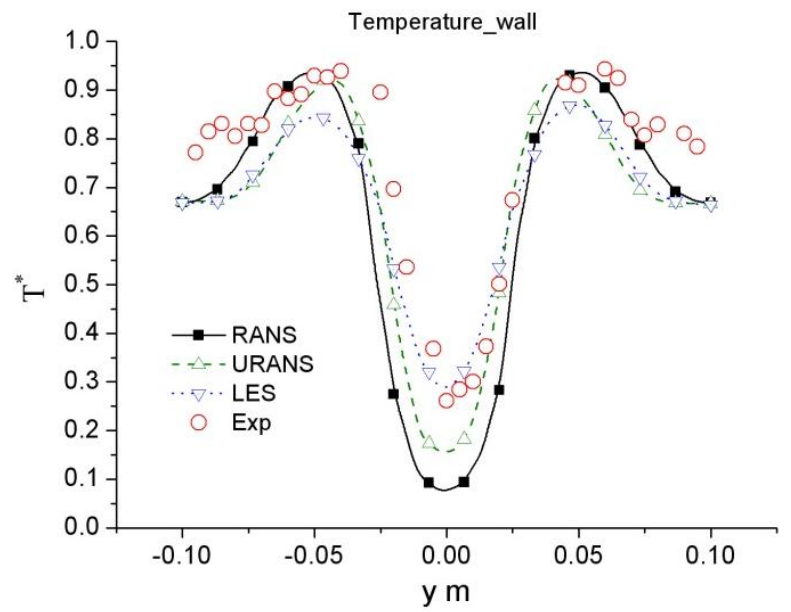

(b) Temperature along the near-wall line

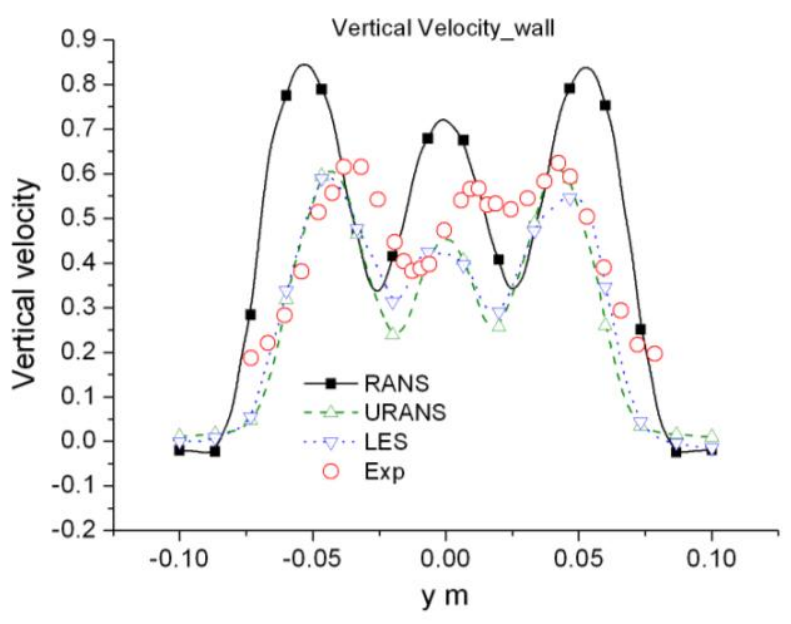

(d) Vertical Velocity along the near-wall line 


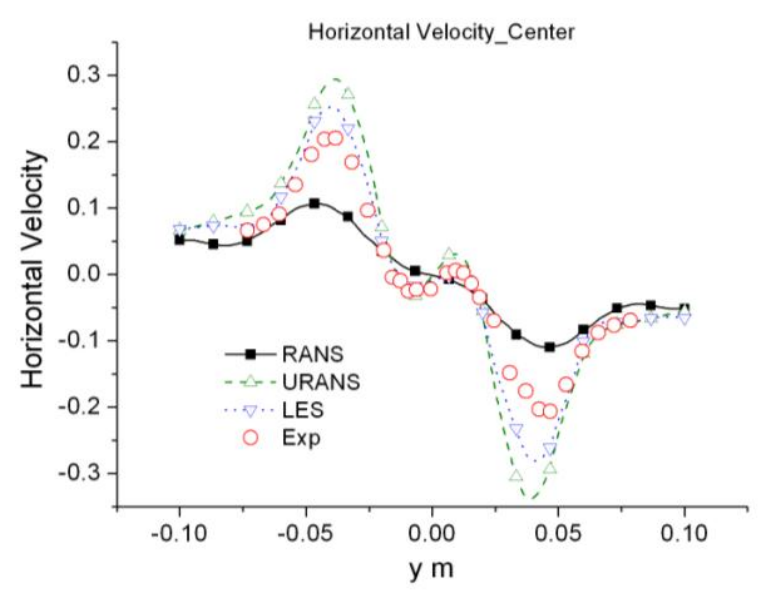

(e) Horizontal Velocity along the centerline

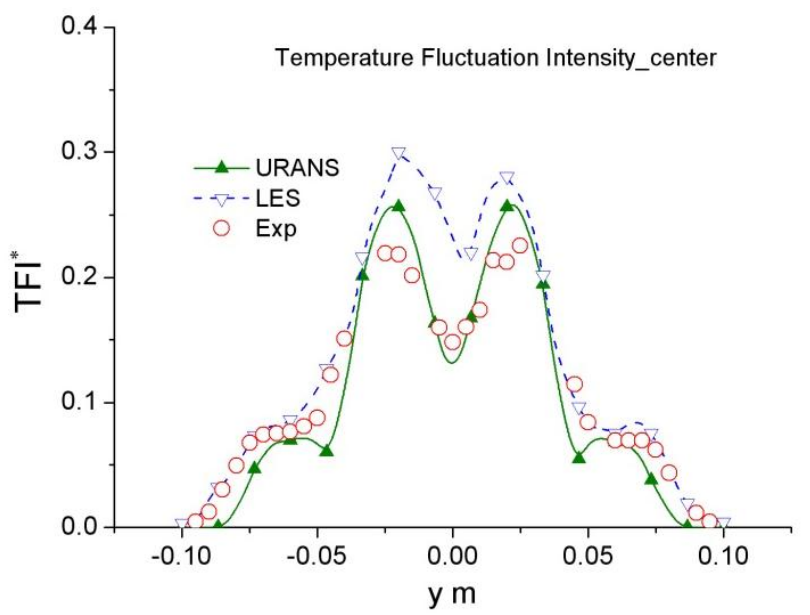

(g) TFI along the centerline

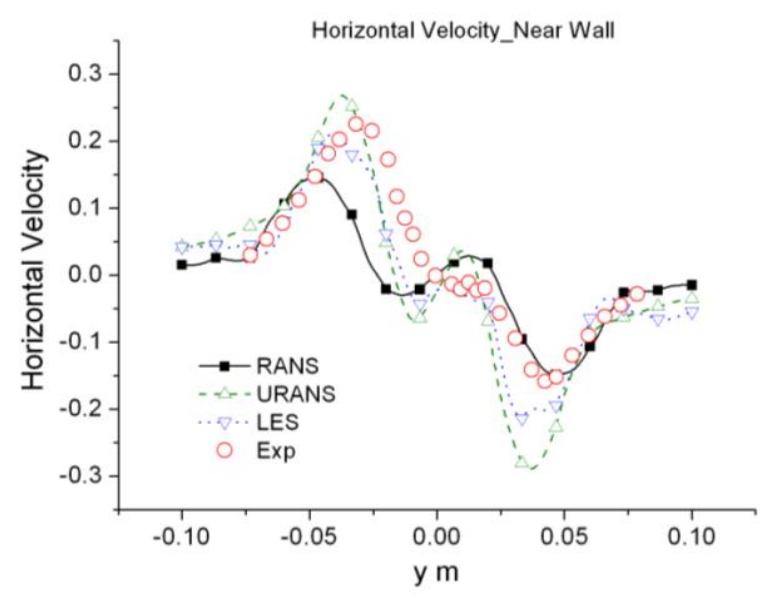

(f) Horizontal Velocity along the near-wall line

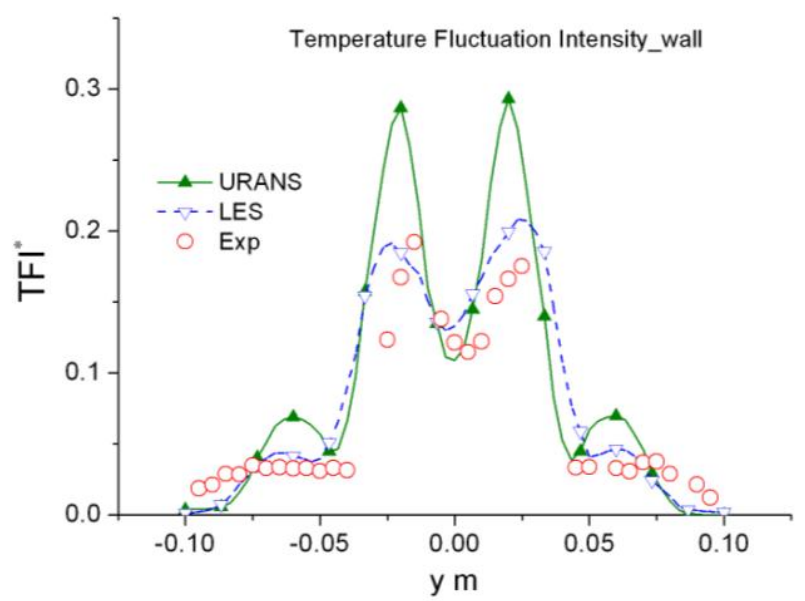

(h) TFI along the near-wall line

Figure 4-5. Temperature, Velocity component and Temperature Fluctuation Intensity on the Centerline and the near-wall with RANS, URANS and LES Models

Figure 4-6 shows the temperature fluctuation intensity distribution in a direction normal to the wall surface. The decay of the TFI in structure is in good agreement with the experiment. Based on Figures 4.5 and 4.6, LES model with conjugate heat transfer can model the thermal interaction between the fluid and the structure well. 


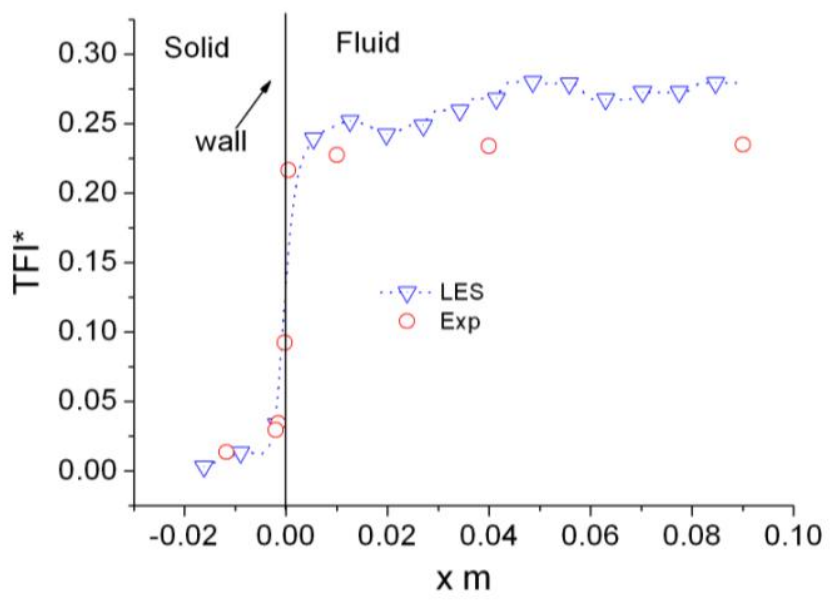

Figure 4-6. Temperature Fluctuation Intensity distribution in Normal Direction to Wall Surface

\section{Conclusions}

This paper presented the predictions of the PLAJEST experiment with sodium coolant using STAR-CCM+. The influence of boundary conditions and conjugate heat transfer were studied. This investigation shows the issues related to using symmetry boundary conditions, including the formation of asymmetric vortices that are non-physical. It also demonstrated that using an outlet boundary along the entire outer perimeter, e.g. both sides and the top, yields the most physical results without inducing numerical instabilities. Employing conjugate heat transfer significantly improves the accuracy of predicting the temperature fluctuation intensity near the wall, but does not influence the predictions in the central plane significantly. The preliminary results of LES and URANS, with outlet boundaries and conjugate heat transfer, exhibited reasonably good agreement with experimental results for temperature fluctuation intensity, as well as the average temperature and vertical velocity components at the measurement locations.

\section{Acknowlegement}

The submitted manuscript has been created by UChicago Argonne, LLC, Operator of Argonne National Laboratory ("Argonne"). Argonne, a U.S. Department of Energy Office of Science laboratory, is operated under Contract No. DE-AC02-06CH11357. The U.S Government retains for itself, and others acting on its behalf, a paid-up nonexclusive, irrevocable worldwide license in said article to reproduce, prepare derivate works, distribute copies to the public, and perform publicly and display publicly, by or on behalf of the Government. Argonne National Laboratory's work was supported by the US DOE Office of Nuclear Energy under contract number DE-AC02-06CH11357.

\section{References}

M. Wakamatsu, H. Nei, K. Hashiguchi, Attenuation of temperature fluctuations in thermal striping, J. Nucl. Sci. Technol. 32 (8) (1995) 752-762. 
H.Y. Nam, J.M. Kim, Thermal striping experimental data. Internal report, LMR/IOC-ST-002-04Rev.0/04, KAERI, 2004.

S.-K. Choi, H.Y. Nam, M.H. Wi, S.-O. Kim, J.C. Jo, H.K. Choi, Evaluation of turbulence models for thermal striping in a triple-jet, in: Proceedings of the ASME Pressure Vessels and Piping Conference, vol. 4, 2005, pp. 867-873.

S.-K. Choi, S.-O. Kim, Evaluation of turbulence models for thermal striping in a triple jet, ASME: J. Pressure Vessel Technol. 129 (4) (2007) 583-592.

A. Manera, H.-M. Prassera, R. Lechner, T. Frank, Toward the prediction of temperature fluctuations by means of steady RANS for the estimation of thermal fatigue, in: $13^{\text {th }}$ International Topical Meeting on Nuclear Reactor Thermal Hydraulics (NURETH-13), 27 September-2 October, Kanazawa City, Ishikawa, Japan, 2009.

Y.Q. Yu, B.H. Yan, X. Cheng, H.Y. Gu Simulation of turbulent flow inside different subchannels in tight lattice bundle, Ann. Nucl. Energy, 38 (2011), pp. 2363-2373

X. Cheng, Y.Q. Yu, Local thermal-hydraulic behaviour in tight 7-rod bundles, Nuclear Engineering and Design, Volume 239, Issue 10, October 2009, Pages 1944-1955

D. S. Wood, "Proposal for design against thermal striping", Nuclear Energy, No.6, pp.433-437, 1980.

J. E. Brunings, "LMFBR thermal-striping evaluation”, Interim report, Research Project 1704-11, prepared by Rockwell International Energy Systems Group, EPRI-NP-2672, 1982.

C. Betts, C. Bourman and N. Sheriff, "Thermal striping in liquid metal cooled fast breeder reactors", NURETH-2, Vol.2, pp.1292-1301, 1983.

S. Moriya, S. Ushijima, N. Tanaka, S. Adachi and I. Ohshima, "Prediction of Thermal Striping in Reactors", Int. Conf. Fast Reactors and Related Fuel Cycles, Vol.1, pp.10.6.1-10.6.10, 1991.

A. Tokuhiro, N. Kimura, J. Kobayashi and H. Miyakoshi, "An investigation on convective mixing of two buoyant, quasi-planar jets with a non-buoyant jet in-between by ultrasound Doppler velocimetry", ICONE-6, ICONE-6058, 1998.

D. Tenchine and H. Y. Nam, "Thermal hydraulics of co-axial sodium jets", Am. Inst. Chem. Engrs. Symp. Ser. 83, No.257, pp.151-156, 1987.

D. Tenchine and J. P. Moro, "Experimental and numerical study of coaxial jets", NURETH-8, Vol. 3, pp.1381-1387, 1997.

E. Merzari, "On the numerical simulation of thermal striping in the upper plenum of a fast reactor”, ICAPP 2010, San Diego, CA, USA, June 2010.

Lomperski, S. et al., 2012. PIV Accuracy and Extending the Field of View for Validation of Multi-Scale CFD Tools. In: Advances in Thermal Hydraulics 2012 (ATH '12), San Diego, CA, USA, November.

A. Tokuhiro, N. Kimura and H. Miyakoshi, "An experimental investigation on thermal striping. Part I: Mixing of a vertical jet with two buoyant heated jets as measured by ultrasound Doppler velocimetry”, NURETH-8, Vol. 3, pp.1712-1723, 1997. 
A. Tokuhiro and N. Kimura, "An experimental investigation on thermal striping. Mixing phenomena of a vertical non-buoyant jet with two adjacent buoyant jets as measured by ultrasound Doppler velocimetry", Nucl. Eng. Design, 188, pp.49-73, 1999.

N. Kimura, A. Tokuhiro and H. Miyakoshi, “An experimental investigation on thermal striping. Part II: Heat transfer and temperature measurement results", NURETH-8, Vol. 3, pp.1724-1734, 1997.

N. Kimura, H. Kamide, P. Emonot and K. Nagasawa, "Study on Thermal Striping Phenomena in Triple-Parallel Jet - Investigation on Non-Stationary Heat Transfer Characteristics Based on Numerical Simulation -", NURETH-12, Log Number: 117, Pittsburgh, Pennsylvania, U.S.A. September 30-October 4, 2007.

N. Kimura, H. Miyakoshi and H. Kamide, "Experimental Investigation on Transfer Characteristics of Temperature Fluctuation from Liquid Sodium to Wall in Parallel Triple-jet", Int. J. Heat and Mass Transfer, 50, pp.2024-2036, 2007.

N. Kimura, H. Miyakoshi, H. Ogawa, H. Kamide, Y. Miyake and K. Nagasawa, "Study on Convective Mixing Phenomena in Parallel Triple-Jet along Wall - Comparison of Temperature Fluctuation Characteristics between Sodium and Water -", NURETH-11, Paper: 427, Avignon, France, October 2-6, 2005.

N. Kimura, H. Kamide, P. Emonot and K. Nagasawa, "Study on Thermal Striping Phenomena in Triple-Parallel Jet - Transfer Characteristics of Temperature Fluctuation in Sodium and Water based on Conjugated Numerical Simulation -", NUTHOS-7, Seoul, Korea, October 5-9, 2008. 